\title{
NOTES
}

\section{Metachromasy in Polymer Films. Changes in the Absorption Spectrum of Methylene Blue in Nafion Films by Hydration}

\author{
Soichi Otsuki and Kimihiro AdachI \\ Government Industrial Research Institute, Osaka, 1-8-31 Midorigaoka, Ikeda 563, Japan
}

(Received April 23, 1993)

\begin{abstract}
KEY WORDS Metachromasy / Methylene Blue / Nafion Film / Absorption Spectrum / Relative Humidity / Hydration /
\end{abstract}

Spectral changes of dyes occur when the concentration of the dyes increases in solution or when polyelectrolytes are added to the solution of the dyes. This color change, which is not correlated with the change of the chemical structure, is termed metachromasy. Variability in color of dyes may be also encountered in polymer films. The absorption spectrum of dyes is affected by the microenvironment in the polymer, which can be altered by the sorption of gas or liquid. Little work has been done on the metachromasy in polymer films in spite of these possibilities. Recently, the color change of dye-doped polymer films has been studied in order to use it for optical humidity detection. ${ }^{1-4}$ In these studies, however, the response of the humiditysensitive film alone was described without details of the humidity dependence of the absorption spectrum of the dyes in the polymer films.

This is the first straightforward investigation on the metachromasy in polymer films. The color change of methylene blue (MB) in a perfluorinated polymer film was studied. The hydration state of the film was altered by equilibration with air currents of various relative humidity $(\mathrm{RH})$. The absorption spectrum of the film containing $\mathrm{MB}$ was measured in various hydration states. The nature of the dye in the film and the interaction of the dye and the polymer were discussed.

\section{EXPERIMENTAL}

Nafion (1100 equiv. wt.; water-alcohol solution; $5 \% \mathrm{w} / \mathrm{v}$; Aldrich) and MB (hydrochloride; extra pure; Ishizu Pharmaceutical) were used as received. Nafion films containing MB for study were prepared by two different protocols. A glass plate coated with indium tin oxide (ITO) was used as a substrate for MB-impregnated films, because Nafion films cast directly on glass are easily removed by immersion in water. The $5 \% \mathrm{w} / \mathrm{v}$ Nafion solution was diluted 4-fold with ethanol. $0.05 \mathrm{ml}$ of the resulting solution was deposited on an ITO-coated glass plate $(30 \times 9.5 \mathrm{~mm})$, air-dried at room temperature, and washed with water. The Nafion film thus obtained was immersed in $10 \mathrm{ml}$ of a $5 \times 10^{-4} \mathrm{M}$ aqueous solution of $\mathrm{MB}$ for a measured time, washed with water, and allowed to dry in room air. For an MB-mixed film, MB was dissolved in ethanol-water $(2: 1 \mathrm{v} / \mathrm{v})$ at a concentration of $1.89 \times 10^{-3} \mathrm{M}$ and mixed with the same volume of the $5 \% \mathrm{w} / \mathrm{v}$ Nafion solution. $0.1 \mathrm{ml}$ of the solution thus obtained was cast on a glass plate $(30 \times 9.5 \mathrm{~mm})$ and the solvent allowed to evaporate. The resulting film contained 0.038 mol kg-1 of MB. Absorption spectrum was measured on a JASCO UVIDEC-1 double- 
beam spectrometer. Absorbance measurements under the condition of desired $\mathrm{RH}$ were made at $25^{\circ} \mathrm{C} \pm 0.2^{\circ} \mathrm{C}$ as described elsewhere. ${ }^{5}$

\section{RESULTS AND DISCUSSION}

The spectral nature of $\mathrm{MB}$ in solution is well-known. The spectrum of MB in water is made up by the superposition of two bands: one with a maximum at $656.5 \mathrm{~nm}$ due to the monomer of the monocation form $\left(\mathrm{MB}^{+}\right)$is more prominent in dilute solutions; the second with a maximum at $600 \mathrm{~nm}$ due to the dimer $\left(\left(\mathrm{MB}^{+}\right)_{2}\right)$ is stronger in concentrated solutions. ${ }^{6}$ In acidic solutions the dication form $\left(\mathrm{MBH}^{2+}\right)$ predominates, whose spectrum consists of a large peak at $742 \mathrm{~nm}$, a medium peak at about $680 \mathrm{~nm}$, and a shoulder at about $610 \mathrm{~nm}^{7}$

MB was incorporated in a Nafion film by immersing the film in an aqueous MB solution. The MB concentration in the Nafion films apparently increases with increasing immersion time. The absorption spectrum of MBimpregnated Nafion films was measured after the films were dipped in water and equilibrated for more than $20 \mathrm{~min}$. The dip of the film in water makes the fully hydrated state of the film. Figure 1 shows the visible absorption spectra of Nafion films immersed in the MB solutions for three different times. With the immersion

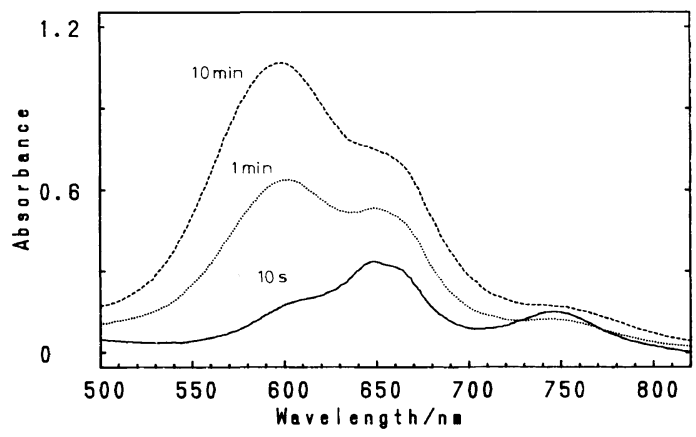

Figure 1. Absorption spectra of Nafion films impregnated with $\mathrm{MB}$ by immersing the films in aqueous MB solutions for $10 \mathrm{~s}, 1 \mathrm{~min}$, and $10 \mathrm{~min}$. The films were dipped in water through the measurement. for $10 \mathrm{~s}$ in the dye solution, an absorption peak at $649 \mathrm{~nm}$ with a shoulder at about $660 \mathrm{~nm}$ appears. This absorption is probably due to $\mathrm{MB}^{+}$. With increasing immersion time, a shoulder at about $600 \mathrm{~nm}$ develops. With the immersion for $10 \mathrm{~min}$ an absorption peak becomes pronounced at $598 \mathrm{~nm}$, which corresponds to $\left(\mathrm{MB}^{+}\right)_{2}$. The effect of the $\mathrm{MB}$ concentration on the absorption spectrum is the same as in solution. The existence of $\mathrm{MB}^{+}$ and $\left(\mathrm{MB}^{+}\right)_{2}$ has been established for $\mathrm{MB}$ impregnated Nafion films. ${ }^{8,9}$ Solid Nafion has a structure like an inverse micelle; the ionic part of Nafion is arranged in non-spherical clusters within the fluorocarbon phase. ${ }^{10}$ Absorbed water and dye molecules reside in the ionic clusters. Water molecules in the ionic clusters have a similar nature as in solution, although they are weakly hydrogen-bonded. ${ }^{11}$ Consequently the formation of dimers is expected to take place within Nafion films similarly as in solution. In addition, an absorption band due to $\mathrm{MBH}^{2+}$ appears at $747 \mathrm{~nm}$. With the immersion for $10 \mathrm{~s}$ this band is prominent. However, no information is described in the literature concerning the formation of $\mathrm{MBH}^{2+}$ in Nafion films. It seems that with decreasing $\mathrm{MB}$ concentration the interaction of MB molecules with sulfonic acid groups of Nafion becomes stronger, which results in the increase of $\mathrm{MBH}^{2+}$.

The hydration state of Nafion films can be altered by changing ambient humidity, at which the films adsorb the equilibrium amount of water vapor. Figures 2(a)-(c) show the visible absorption spectra of MB-impregnated Nafion films, which were recorded at different RHs. Figure 2(a) is the result for the film with an immersion time of $10 \mathrm{~s}$. At $0 \% \mathrm{RH}$ there are two peaks at 682 and $752 \mathrm{~nm}$ and a shoulder at about $620 \mathrm{~nm}$, which correspond to $\mathrm{MBH}^{2+}$. Because water molecules are excluded from the ionic cluster at $0 \% \mathrm{RH}$, the interaction between MB molecules and sulfonic acid groups of Nafion is strong without interference of water molecules. Therefore, it is predicted that a lot 


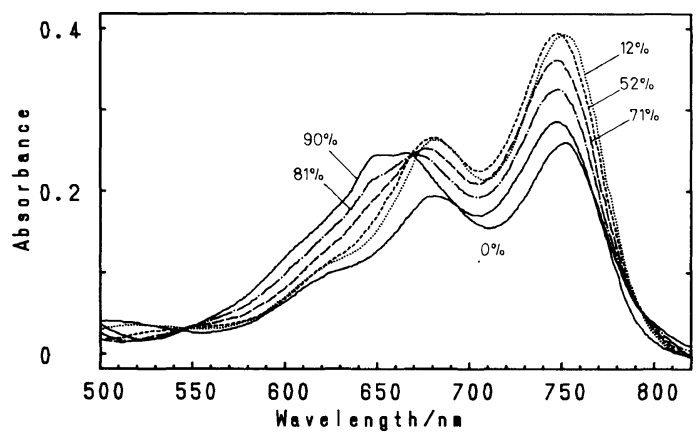

(a)

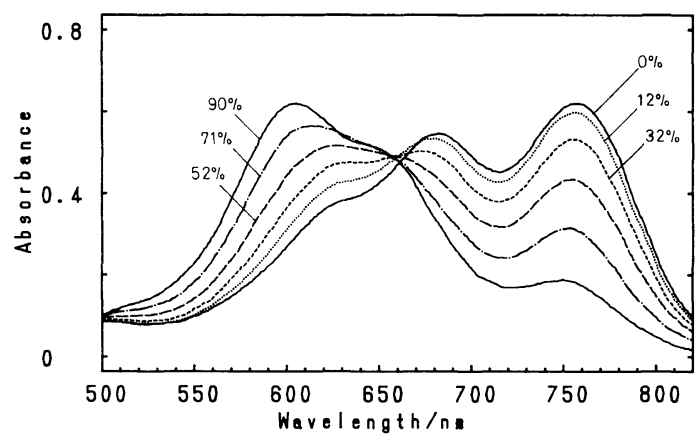

(b)

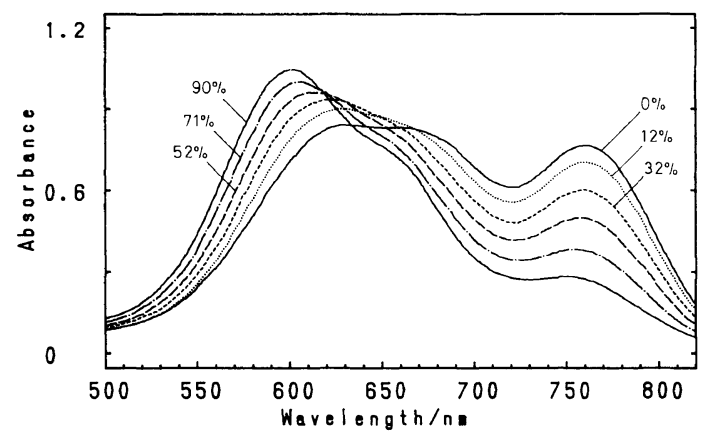

(c)

Figure 2. Absorption spectra of MB-impregnated Nafion films measured at varying RHs. Nafion films were impregnated with $\mathrm{MB}$ by immersing the films in aqueous MB solutions for $10 \mathrm{~s} \mathrm{(a),} 1 \mathrm{~min}(\mathrm{~b})$, and $10 \mathrm{~min}$ (c).

of $\mathrm{MB}$ molecules are protonated to form $\mathrm{MBH}^{2+}$ at $0 \% \mathrm{RH}$. When RH increases to $12 \%$ the bands due to $\mathrm{MBH}^{2+}$ abruptly develop without change in their proportion. As evidenced below, $\mathrm{MBH}^{2+}$ changes to the trication form $\left(\mathrm{MBH}_{2}^{3+}\right)$ at $0 \% \mathrm{RH}$. It seems that the MB concentration is so low with the immersion for $10 \mathrm{~s}$ that one MB molecule interacts with more than one sulfonic acid groups (Figure 3(a)). The peaks at 682 and $752 \mathrm{~nm}$ shift slightly to a shorter wavelength with increasing RH but their intensity scarcely changes in the range from $12 \%$ to $52 \% \mathrm{RH}$. Above $52 \% \mathrm{RH}$ new peaks develop at 649 and $665 \mathrm{~nm}$, which correspond to $\mathrm{MB}^{+}$. Concomitantly, the peaks at 682 and $752 \mathrm{~nm}$ are diminished. The amount of water present in the ionic cluster apparently increases with RH. As a result, the interaction between $\mathrm{MB}$ molecules and sulfonic acid groups of Nafion becomes weak because the MB molecules are solvated with water. Thus, the deprotonation proceeds as $\mathrm{RH}$ increases. At $90 \% \mathrm{RH}$ the peaks at 649 and $665 \mathrm{~nm}$ due to $\mathrm{MB}^{+}$become large but the peak at $752 \mathrm{~nm}$ still remains. This indicates that most of $\mathrm{MB}$ monocation exists as monomers and a lot of $\mathrm{MBH}^{2+}$ is also present in the ionic cluster (Figure 3(b)).

Figure 2(b) shows the visible absorption spectra for the film with an immersion time of $1 \mathrm{~min}$. At $0 \% \mathrm{RH}$ there are two peaks at 682 and $757 \mathrm{~nm}$ and a shoulder at about $620 \mathrm{~nm}$, which are characteristic of $\mathrm{MBH}^{2+}$. The relative intensity of the bands at 682 and $620 \mathrm{~nm}$ is larger than that for the $10 \mathrm{~s}$-immersed film. This is probably because the peaks due to the monocation forms are superposed on these bands. It can be therefore said that many MB molecules are protonated but some MB molecules exist as the monocation forms (Figure 3(c)). It is supposed that the number of $\mathrm{MB}$ molecules in the ionic cluster is comparable to that of sulfonic acid groups. As RH increases, the shoulder at about $620 \mathrm{~nm}$ increases and shifts to a shorter wavelength. Concomitantly, the peaks at 682 and $757 \mathrm{~nm}$ are diminished. At $90 \% \mathrm{RH}$ a peak at $605 \mathrm{~nm}$ due to $\left(\mathrm{MB}^{+}\right)_{2}$ predominates. Because the $\mathrm{MB}$ concentration is sufficiently high, many $\mathrm{MB}$ molecules exist as dimers in the ionic cluster (Figure 3(d)).

Figure 2(c) shows the visible absorption 


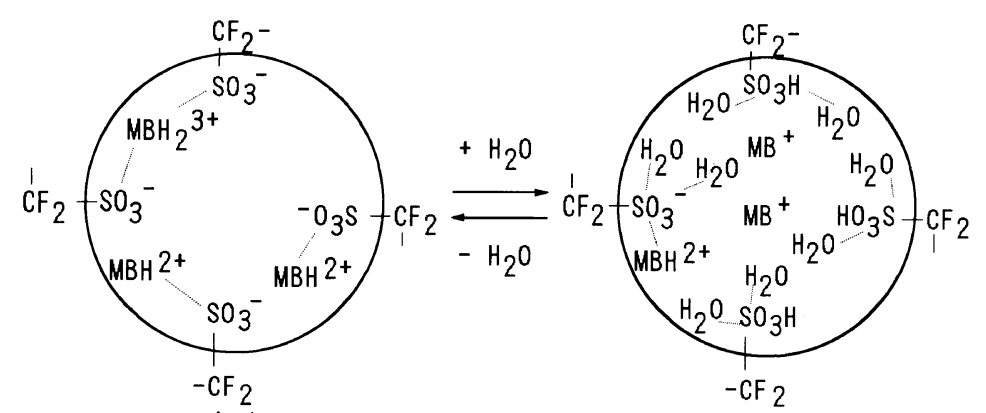

(a)

(b)

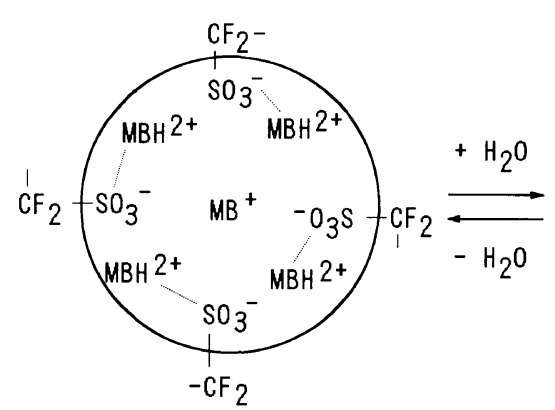

(c)

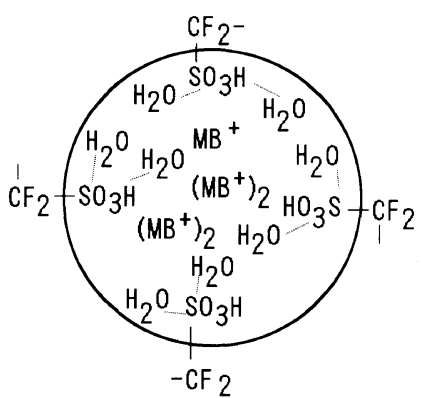

(d)

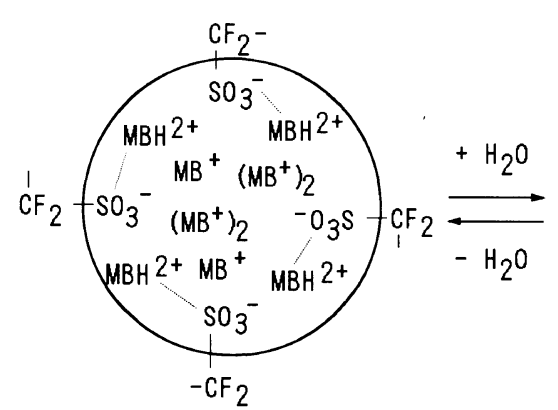

(e)

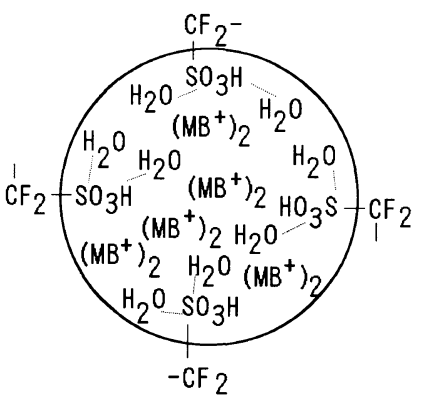

(f)

Figure 3. Effect of hydration on the interaction between $M B$ molecules and sulfonic acid groups in the ionic cluster of Nafion containing different MB concentrations. The number of MB molecules is less (a, b), almost the same (c, d), and greater (e, f) than that of sulfonic acid groups.

spectra for the film with an immersion time of $10 \mathrm{~min}$. The spectrum recorded at $0 \% \mathrm{RH}$, which consists of three main bands at 620,670 , and $761 \mathrm{~nm}$, resembles to the spectra recorded at $32 \%$ and $52 \% \mathrm{RH}$ for the $1 \mathrm{~min}$-immersed film. The situation at $0 \% \mathrm{RH}$ is similar to that in the medium- $\mathrm{RH}$ region for the $1 \mathrm{~min}$ immersed film. Consequently, it is predicted that $\left(\mathrm{MB}^{+}\right)_{2}, \mathrm{MB}^{+}$, and $\mathrm{MBH}^{2+}$ are present at the same time. The peak at $761 \mathrm{~nm}$ is ascribed to $\mathrm{MBH}^{2+}$. The peaks at 620 and $670 \mathrm{~nm}$ are interpreted as unresolved peaks resulting from the superposition of the bands due to $\left(\mathrm{MB}^{+}\right)_{2}$ and $\mathrm{MB}^{+}$, respectively, on the bands due to $\mathrm{MGH}^{2+}$. Probably the number of $\mathrm{MB}$ molecules is fairly greater than that of sulfonic acid groups. In addition, it is expected that the number of sulfonic acid groups that are capable 


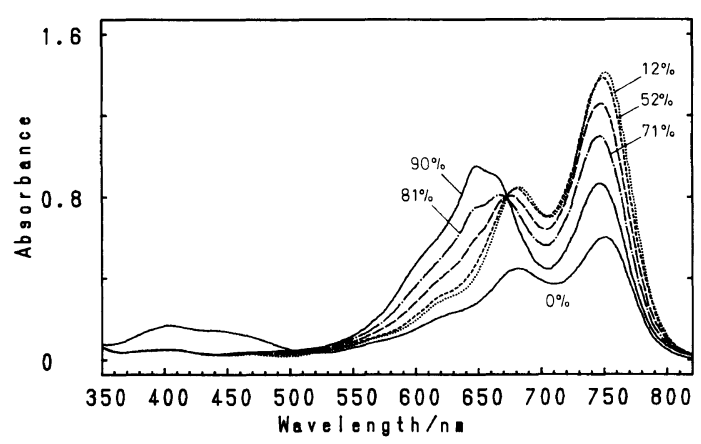

Figure 4. Absorption spectra of an MB-mixed Nafion film measured at varying RHs.

of interacting with $\mathrm{MB}$ molecules is somewhat less than its total number, $0.91 \mathrm{~mol} \mathrm{~kg}^{-1}$. Hence, many MB molecules remain unprotonated and some of them exist as dimers even in the completely dry state (Figure 3(e)). As $\mathrm{RH}$ increases, the peak at $620 \mathrm{~nm}$ increases and shifts to a shorter wavelength. Concomitantly, the peaks at 670 and $761 \mathrm{~nm}$ are diminished. At $90 \% \mathrm{RH}$ the peak at $602 \mathrm{~nm}$ due to $\left(\mathrm{MB}^{+}\right)_{2}$ predominates. The relative intensity of this peak is larger than that for the 1 min-immersed film. It seems that the dimer formation occurs more readily because of the higher concentration of MB (Figure 3(f)).

The same measurement was made for an MB-mixed Nafion film in order to check the effect of the method of film preparation. Figure 4 shows the visible absorption spectra of an MB-mixed Nafion film, which were recorded similarly to MB-impregnated films. At $0 \% \mathrm{RH}$ there are peaks corresponding to $\mathrm{MBH}^{2+}$ at 682 and $751 \mathrm{~nm}$. At $90 \% \mathrm{RH}$ bands due to $\mathrm{MB}^{+}$ get prominent at 649 and $664 \mathrm{~nm}$. Thus, the spectral change with $\mathrm{RH}$ quite resembles that observed for the $10 \mathrm{~s}$-immersed film. This is possibly because the MB concentrations of these two films are almost the same. In addition, at $0 \% \mathrm{RH}$ there are broad peaks at about 400 and $450 \mathrm{~nm}$. Similar peaks have been reported for $\mathrm{MB}$ in $78 \%$ sulfuric acid $^{7}$ but their assignment has not been made. These peaks disappear but the bands due to $\mathrm{MBH}^{2+}$ increase with increasing $\mathrm{RH}$ from $0 \%$ to $12 \%$.
This reveals that the peaks at about 400 and $450 \mathrm{~nm}$ correspond to $\mathrm{MBH}_{2}^{3+}$. A similar increase of the bands due to $\mathrm{MBH}^{2+}$ was observed for the $10 \mathrm{~s}$-immersed film as described above. Therefore, it is clear that $\mathrm{MBH}_{2}^{3+}$ is also formed for the $10 \mathrm{~s}$-immersed film.

\section{CONCLUSIONS}

Metachromasy of MB in Nafion films was thoroughly investigated. When the films are fully hydrated, $\mathrm{MB}^{+}$and $\left(\mathrm{MB}^{+}\right)_{2}$ are formed in the films similarly as in solution. In addition, $\mathrm{MBH}^{2+}$ also exists and increases with decreasing MB concentration. The hydration state of Nafion films is altered by equilibration with air streams of different RHs. At a low MB concentration $\mathrm{MBH}_{2}^{3+}$ together with $\mathrm{MBH}^{2+}$ is formed in the low $\mathrm{RH}$ region, whereas both $\mathrm{MB}^{+}$and $\mathrm{MBH}^{2+}$ are the main species in the high $\mathrm{RH}$ region. It seems that the number of MB molecules is fairly less than that of sulfonic acid groups. At a high $\mathrm{MB}$ concentration $\mathrm{MB}^{+}$, $\left(\mathrm{MB}^{+}\right)_{2}$, and $\mathrm{MBH}^{2+}$ are present in the low RH region, whereas $\left(\mathrm{MB}^{+}\right)_{2}$ predominates in the high $\mathrm{RH}$ region. This is probably because the number of $\mathrm{MB}$ molecules is fairly higher than that of sulfonic acid groups. Because the interaction between MB molecules and sulfonic acid groups is strong in the dry state, dye cations with more than one positive charges are formed. This interaction becomes weak owing to hydration of sulfonic acid groups as the amount of water in the films increases.

\section{REFERENCES}

1. S. Muto, A. Fukasawa, T. Ogawa, M. Morisawa, and H. Ito, Jpn. J. Appl. Phys., 29, L1023 (1990).

2. K. Wang, K. Seiler, J. P. Haug, B. Lehmann, S. West, K. Hartman, and W. Simon, Anal. Chem., 63, 970 (1991).

3. Y. Sadaoka, M. Matsuguchi, and Y. Sakai, J. Electrochem. Soc., 138, 614 (1991).

4. R. A. McGill, M. S. Paley, and J. M. Harris, Macromolecules, 25, 3015 (1992).

5. S. Otsuki and K. Adachi, J. Appl. Polym. Sci., 48, 
1557 (1993).

6. E. Rabinowitch and L. F. Epstein, J. Am. Chem. Soc., 63, 69 (1941).

7. G. N. Lewis and J. Bigeleisen, J. Am. Chem. Soc., 65, 1144 (1943).

8. S. Kuwabata, J. Nakamura, and H. Yoneyama, J.
Electroanal. Chem., 261, 363 (1989).

9. A. R. Guadalupe, K. E. Liu, and H. D. Abruna, Electrochim. Acta, 36, 881 (1991).

10. H. L. Yeager and A. Steck, J. Electrochem. Soc., 128, 1880 (1981).

11. M. Falk, Can. J. Chem., 58, 1495 (1980). 